\title{
Editorial
}

Gérard S. Chetrite ${ }^{1}$ / Bruno Fève ${ }^{2}$

\section{Preface to special issue on "Liver Metabolic Diseases and Hepatocellular Carcinoma: New Hormonal and Clinical Insights"}

\author{
${ }^{1}$ Assistance Publique-Hôpitaux de Paris (AP-HP), Service d'Endocrinologie et des Maladies de la Reproduction, Hôpitaux Uni- \\ versitaires Paris-Sud, CHU Bicêtre, Faculté de Médecine Paris-Sud, Université Paris-Saclay, Institut Hospitalo-Universitaire \\ ICAN, Le Kremlin Bicêtre, France, E-mail: gerard.chetrite@u-psud.fr \\ ${ }^{2}$ Service d'Endocrinologie, Hôpital Saint Antoine, INSERM, UMR S 938, Centre de Recherche Saint-Antoine, Université Pierre \\ et Marie Curie, Faculté de Médecine, Institut Hospitalo-Universitaire ICAN, Paris, France, E-mail: bfeve@free.fr
}

DOI: $10.1515 / \mathrm{hmbci}-2020-2067$

Liver and hepatocyte cells form a complex pivotal human tissue implicated in multiple physiological functions, notably in the detoxification process, in the regulation of lipid and glucide metabolisms, and in energy homeostasis. During recent years, the frequency of liver diseases with hepatic steatosis transformation has greatly increased, at the same time as the epidemic evolution of overweight and obesity worldwide.

In fact, besides the well-known risk factors of liver illness, such as viral hepatitis (B and C) infection and alcohol consumption abuse, new sedentary lifestyle and bad nutritional habits like a diet of junk food with high salt, sugar, and fat, lead to a chronic low-grade inflammatory status, central/visceral android adiposity, insulin resistance, dyslipidemia, type 2 diabetes mellitus, and metabolic syndrome, which provoke hepatic tissue pathogenesis and liver metabolic damage, associated with an unhealthy histological progression through a simple hepatic steatosis, a nonalcoholic steatohepatitis (NASH), a nonalcoholic fatty liver disease (NAFLD) status, then fibrosis, cirrhosis, and eventually to hepatocellular carcinoma (HCC) and hepatic failure. It is observed that NAFLD is the most common liver illness and represents more than $30 \%$ of the whole population, whereas HCC is the third cause of cancer-related death in the world. HCC also possesses a sex-specific preference for men as its incidence is much higher than that for women. What are the different molecular mechanisms involved in the malignant transformation of safe liver to NAFLD and HCC? This important question is yet under scrutiny.

Among the multiple approaches which can initiate and develop liver metabolic diseases, the dysfunction of growth factor pathways, cytokines/hepatokines/myokines/adipokines availability, and/or hormonal signaling are crucial factors in liver diseases. Indeed, these endocrine, paracrine, autocrine and intracrine networks also disturb the crosstalk of the liver with other organs and tissues such as the pancreas, gastrointestinal tract, skeletal muscle, adipose tissue, and neuroendocrine axis.

Furthermore, the control of endocrine modulators, nutritional factors, and obesity-associated diseases is a major goal for managing NAFLD and liver disorders and for finding new therapeutic and clinical perspectives. Moreover, the liver is a peculiar organ by its interesting regenerative capacity to assume, with the presence of hepatic progenitor cells, the partial injury-induced liver regeneration which can participate in treating liver damage.

We are happy to propose to the readers of Hormone Molecular Biology and Clinical Investigation (HMBCI) a very interesting state-of-the-art special issue on "Liver Metabolic Diseases and Hepatocellular Carcinoma: New Hormonal and Clinical Insights" (Volume 41, Issue 1).

This issue has been compiled by leaders in this field, and assembles a group of five reviews and one original article that highlight new important data concerning the capital role of liver crosstalk with different crucial endocrine and/or metabolic tissues, and their pathophysiological outcome, with positive perspectives for future treatments.

The reviews include: a very clever analysis by the Berg team on the behavior of metalloproteinases in the context of NAFLD and fibrosis associated with insulin resistance and modulation of circulating adipokines; the Gilgenkrantz team explains very clearly the concept of liver regeneration and shows how severe steatosis and NASH can interfere with this process. The Lonardo and Lugari team describes very precisely the effects and the interaction of hypothyroidism in the evolution of NAFLD. The Hodson team compares very exhaustively the 
advantages and/or inconveniences of techniques such as in vivo, ex vivo, and in vitro models for studying the development and progression of NAFLD. In an original article, the Sasano team finely determines the effects of estrogens in the development of HCC by analyzing the expression of aromatase and 17 $\beta$-hydroxysteroid dehydrogenase types 1 and 2 in normal and diseased liver such as NASH, hepatitis B, or C virus infections. Finally, the Desbois-Mouthon team presents a very attractive review on the potential mechanisms involved in the development and progression of HCC from a NASH environment.

We express our sincere thanks to the distinguished, internationally renowned scientists, researchers, and clinicians, who kindly accepted to contribute with their expertise to this special issue, providing a precious update on both the growing number of liver metabolic diseases and HCC, with notably potential clinical and therapeutic investigations that could have major clinical consequences affecting the health and well-being of millions of people throughout the world. We trust that the readers of HMBCI will enjoy browsing through and consulting this special issue, hopefully finding answers and, perhaps more pertinently, provoking further questions, to capture the interest and encourage more articles in this field. We look forward to sharing this continuing adventure in the future issues of our journal.

We warmly thank Ms S.Y. MacDonald for the quality of her help in the editorial process. 